IFJPAN-IV-2017-6

\title{
Extra lepton pair emission corrections to Drell-Yan processes in PHOTOS and SANC
}

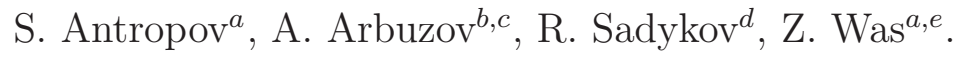 \\ ${ }^{a}$ Institute of Nuclear Physics, Polish Academy of Sciences, ul. Radzikowskiego 152, 31-342 \\ Krakow, Poland; \\ ${ }^{b}$ Bogoliubov Laboratory for Theoretical Physics, JINR, Dubna 141980, Russia; \\ ${ }^{c}$ Dubna State University, Dubna 141982, Russia; \\ ${ }^{d}$ Dzhelepov Laboratory of Nuclear Problems, JINR, Joliot-Curie str. 6, 141980 Dubna, Russia; \\ $e$ Theory Group, Physics Department, CERN, CH-1211, Geneva 23, Switzerland.
}

\begin{abstract}
In the paper we present results for final state emissions of lepton pairs in decays of heavy intermediate states such as Z boson. Short presentations of PHOTOS and SANC algorithms and physics assumptions are given. Numerical distributions of relevance for LHC observables are shown. They are used in discussions of systematic errors in the predictions of pair emissions as implemented in the two programs. Suggestions for the future works are given. Present results confirm, that for the precision of $0.3 \%$ level, in simulation of final state the pair emissions can be avoided. For the precision of $0.1-0.2 \%$, the results obtained with the presented programs should be enough. To cross precision tag of $0.1 \%$, the further work is however required.
\end{abstract}

IFJPAN-IV-2017-6

June 2017 


\section{Introduction}

With the increasing precision of measurements more detailed theoretical calculations are needed for interpretation of results in the language of physics parameters such as masses or couplings of $\mathrm{Z}$ and $\mathrm{W}$ bosons. In the present note, we concentrate on effects and uncertainties related to emission of real lepton pair in association with Drell-Yan processes. Our work is a direct continuation of [1], that is why we will omit many definitions included in that paper. We will concentrate on the effects related to additional pair emissions in decays of heavy bosons, mainly $Z$.

Our main goal is to study the effect of light pair emission $f \bar{f}$ in neutral current DrellYan process $q \bar{q} \rightarrow \gamma / Z \rightarrow \ell^{+} \ell^{-}(f \bar{f})$ for $p p$ collisions at the LHC. We consider the cases $\ell=e, \mu$ and $f=e, \mu$. This effect should be included starting from the second order of QED, i.e. from the $\mathcal{O}\left(\alpha^{2}\right)$ corrections. The typical Feynman diagrams for pair corrections are shown in Fig. 1,

Real pair emission
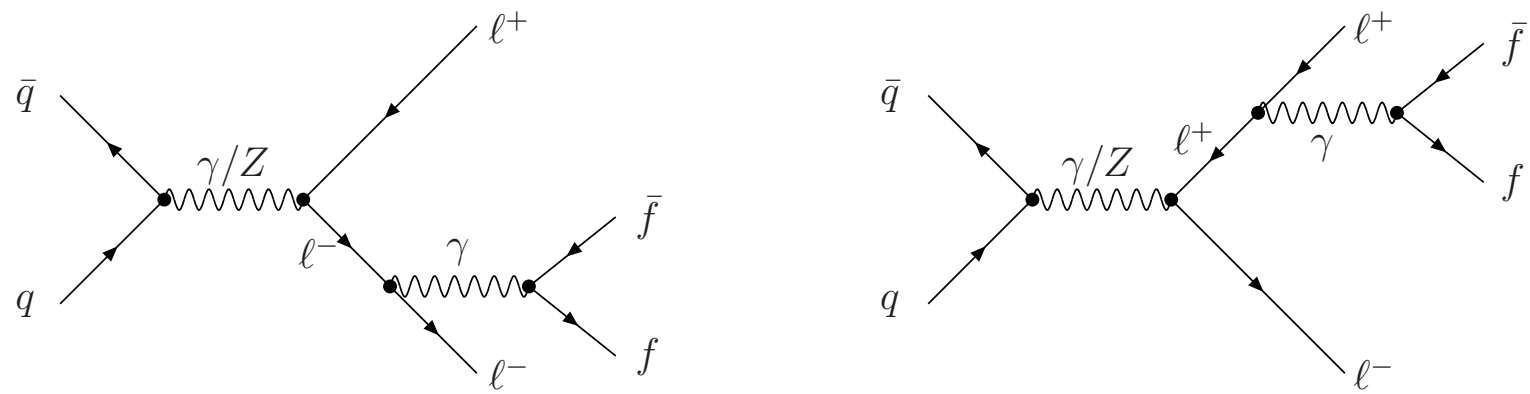

Virtual pair correction

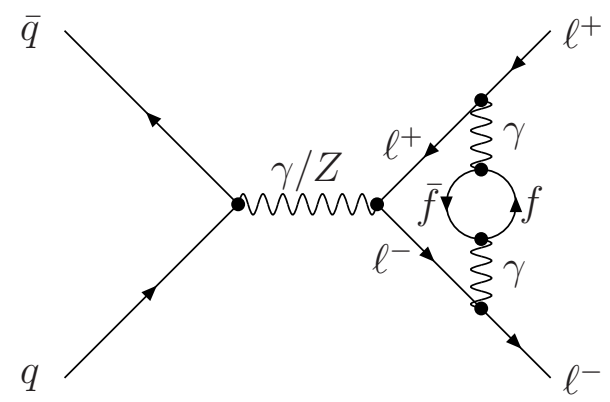

Figure 1: Feynman diagrams for real and virtual pair correction.

The PHOTOS [2 8] and SANC [9 18] Monte Carlo programs use different an approximations for the effect under study. We will show the program features important for effect of pair emissions respectively in Section 2 and 3. The numerical comparison of the results from the two programs and benchmark semi-analytical calculations follows. In Section 4 the definition of our tests distributions is given. Main results are also collected in this section. Section 5 is devoted to the case of mixed pair and photon emissions and summary 
Section 6 closes the paper. Extensive Appendix collects result of our new semi-analytical calculations for pair emissions which is used to obtain numerical results necessary to understand origin of PHOTOS-SANC differences.

\section{Pair corrections in PHOTOS}

The basis of PHOTOS algorithm is of the after-burner type. For the previously generated event, with a certain probability, a decay vertex can be replaced with the one featuring additional photons (similar solution for additional lepton pairs is installed) 8].

For that purpose, PHOTOS uses the exact phase space parametrizations. The best description of its phase-space generation is given in [7]. Case of pair emission is quite analogous and the kinematical configuration for each decay is first deconvoluted into angular parametrization of two body decay into emitter and spectator 1. The corresponding angles, together with extra generated ones, provide parametrization of four body phase space; all necessary phase-space Jacobians are calculated and taken into account. Corresponding algorithm for phase-space is also exact in the case of emission of additional lepton pairs.

It was checked with samples of 100 million events that once matrix element is set to unity, flat four body phase space generation is achieved. This was checked with default test of MC-TESTER [19].

Before matrix element installation, pre-samplers were introduced and checked as well, respectively for collinear, small virtuality and small energy of virtual photon enhancements. For the case of two channels of singularity structure, two pre-samplers are needed. In this case phase space parametrization remains exact. However, when further particles, such as additionally generated photons appear, parametrization of phase-space ceases to be exact. This is due to the matching of Jacobians for distinct generation branches. This non-exactness appear as in multi-photon's emission or in any other case of more than two body decays in PHOTOS operation.

The probability distribution for pair emission is independent from the Born-level matrixelement squared. It is defined by integrand for $\tilde{B}_{f}$ (formula (1) from [20]). Such a formula is valid for the soft pairs emissions but is applied, at present, in PHOTOS Monte Carlo algorithm over the entire phase space. If the energy of the emitted pair is smaller than $\Delta\left(2 m_{f} \ll \Delta \ll \sqrt{s}\right)$ then the formula (11) from [20] is valid too. It was used to check the validity of PHOTOS prediction in the soft region. Agreement at the expected level of few percents of pair effect was found for electrons and muons, and for several choices of maximum energy of emitted lepton pairs.

Further work on matrix element used in PHOTOS can be continued, once tests of the present version are completed. The corresponding task is going to be rather straightforward. The presently used matrix element is calculated in separate program unit directly from the decay products four-vectors. Test, with the help of KORALW [21] Monte Carlo featuring matrix element for $Z$ to four fermions decay, is reported.

${ }^{1}$ The spectator may represent multiple particles. But as corresponding Jacobians for phase space parametrization do not need to be modified we may omit details from our brief presentation. 
Emission of pairs can be simultaneous with emission of real photons. The algorithm can be used in such case as well. The solution is consistent for the leading logarithms with evolution equations. Numerical tests were not performed because pair correction is too small to justify the effort. It was only checked that the variants of algorithm do not lead to numerically sizable effects.

For the virtual correction emulation, the sum rule is used.

\section{Pair corrections in SANC}

In SANC the leading logarithmic approximation (LLA) was applied to take into account the corrections of the orders $\mathcal{O}\left(\alpha^{n} L^{n}\right), n=2,3$. The contribution of pair emission is approximated by the formula (8) from [1], where big logarithms $L\left(m_{\ell}, \mu\right)=\log \left(\mu^{2} / m_{\ell}^{2}\right)$ depends on the lepton mass $m_{\ell}$ and on the factorization scale $\mu$. For the sake of comparison we keep only the term proportional to $\alpha^{2}$ in the above-mentioned formula, i.e. the following expression is used:

$$
\mathcal{D}_{\ell \ell}^{\text {pair }}(y, L)=\left(\frac{\alpha}{2 \pi}(L-1)\right)^{2}\left[\frac{1}{3} P^{(1)}(y)+\frac{1}{2} R^{s}(y)\right]
$$

\section{Setup for comparison and numerical results}

For the comparison we used the same scheme and the values of input parameters as in [1] (eq.(2)). The cut on invariant mass $M\left(\ell^{+} \ell^{-}\right)>50 \mathrm{GeV}$ was imposed.

We define the correction as $\delta^{\text {pair }}=\left(\sigma^{\text {pair }}-\sigma^{\text {Born }}\right) / \sigma^{\text {Born }}$. The results for distribution of invariant mass $M\left(\ell^{+} \ell^{-}\right)$are presented in Fig. 2 2 and Fig. [3 for PYTHIA generated sample of Drell-Yan processes at $14 \mathrm{TeV}$ center of mass energy pp collisions and final state of electron and muon pairs respectively.
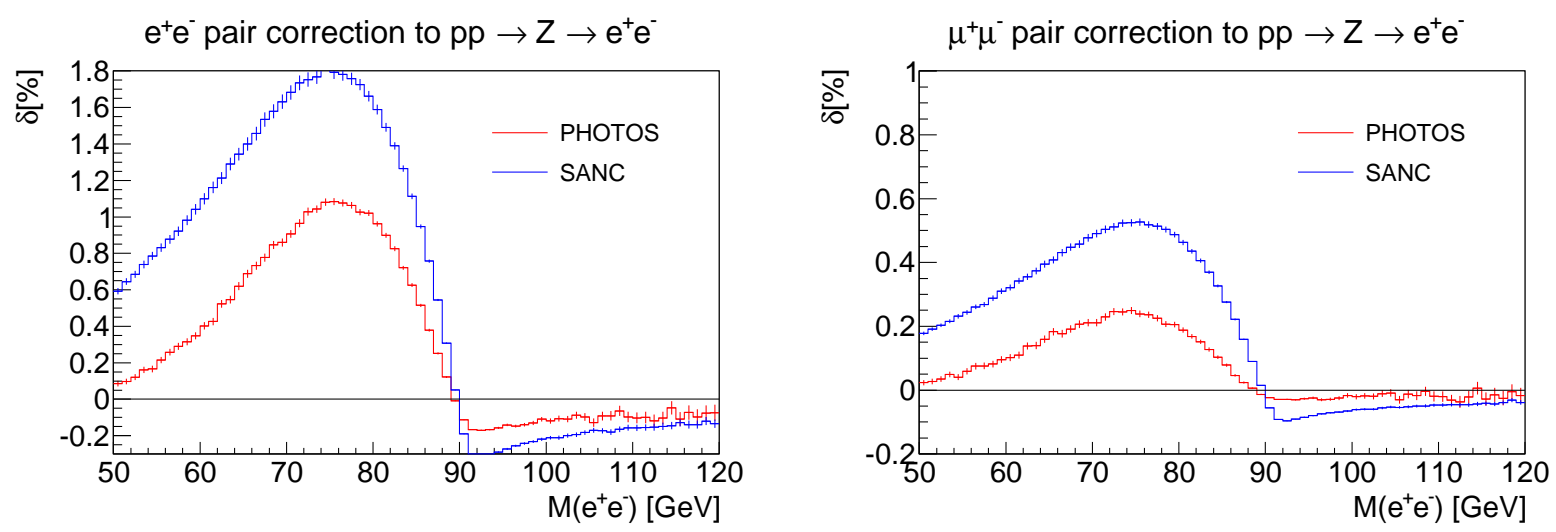

Figure 2: Corrections $\delta$ in $\%$ for invariant mass $M\left(e^{+} e^{-}\right)$distribution in $Z \rightarrow e^{+} e^{-}$decay due to extra $e^{+} e^{-}$(left) or $\mu^{+} \mu^{-}$(right) pair emission. 

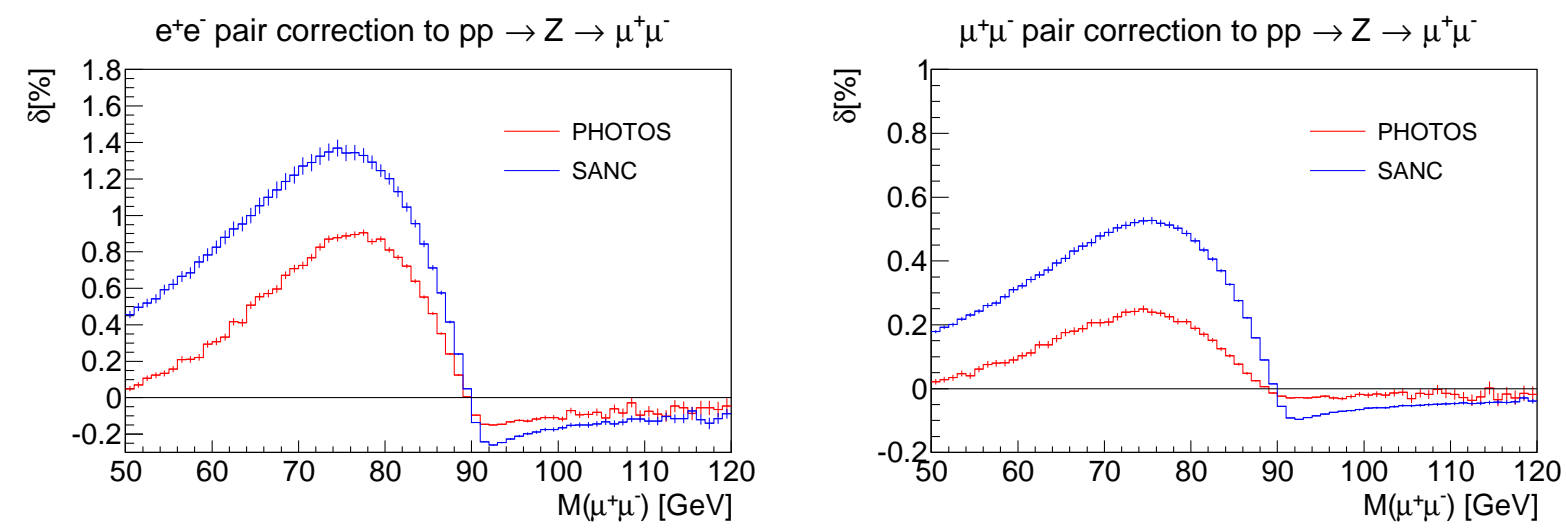

Figure 3: Corrections $\delta$ in \% for invariant mass $M\left(\mu^{+} \mu^{-}\right)$distribution in $Z \rightarrow \mu^{+} \mu^{-}$decay due to extra $e^{+} e^{-}$(left) or $\mu^{+} \mu^{-}$(right) pair emission.

An agreement between pair implementation with the help of PHOTOS and SANC seems not to be sufficient, differences are dominated, as we will see later, by non leading terms and of rather hard pair emission. Let us continue with discussion of results.

The comparison between HORACE 22] and SANC of pair contributions is presented in the Ref. [23]. One can see, that a better agreement was found in this case, but the implementation of pair corrections in HORACE is closer to SANC than to PHOTOS.

Let us stress, that the main purpose of SANC is to control dominant, leading logarithm effects of pairs emission for the sake to supplement systematic error evaluation for observables, where pair effects are comparable to systematic errors of other effects. That is why, non leading terms such as $\ln \frac{\mu}{m_{\mu}} \simeq 6$ may be neglected if they accompany dominant $\ln \frac{\mu}{m_{e}} \simeq 11$ ones. It may be of interest to implement such non-leading terms into SANC and/or PHOTOS.

We start semi-analytical tests. Previous researches in this direction can be found in ref. 24]. Now we will also use formula (5) of ref. 20] (we recall it in Appendix as formula (26)). For its calculation the approximation of factorization for phase space is used, it is universal and applies to initial state pair emissions as well. For technical tests of PHOTOS and for better understanding of the features of differences, the semi analytical calculation was repeated, but with exact parametrization of final state emission phase space. Alternative formula (25) was obtained in Appendix. The numerical tests are summarized in figs. 4 and 5 ,

- We monitor again, as in Figs. 1 and 2, the spectrum of invariant mass for the lepton pair, which is modified by emission of additional pair.

- For results of PHOTOS [8] and for semi-analytical calculation we first generate the sample of events from PYTHIA [25] with initialization summarized in Fig. 12 ,

- In order to complete results for PHOTOS, its algorithm is applied on events generated by PYTHIA. 


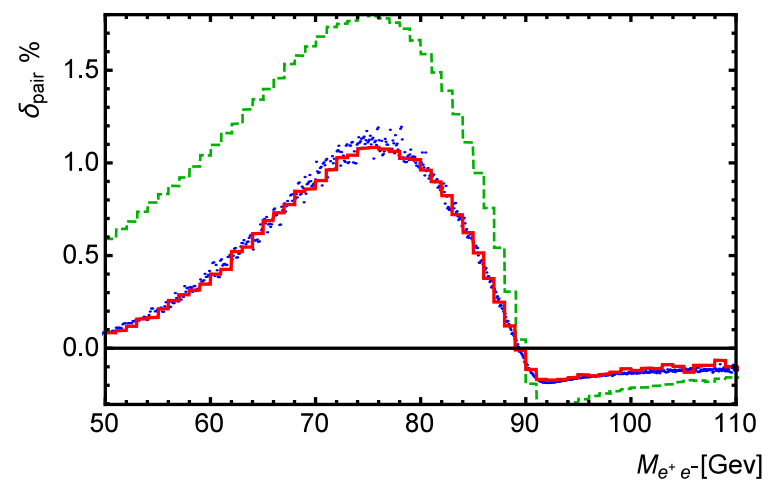

a) Points represent results of simulation by PYTHIA, convoluted bin by bin with our new formula (25).

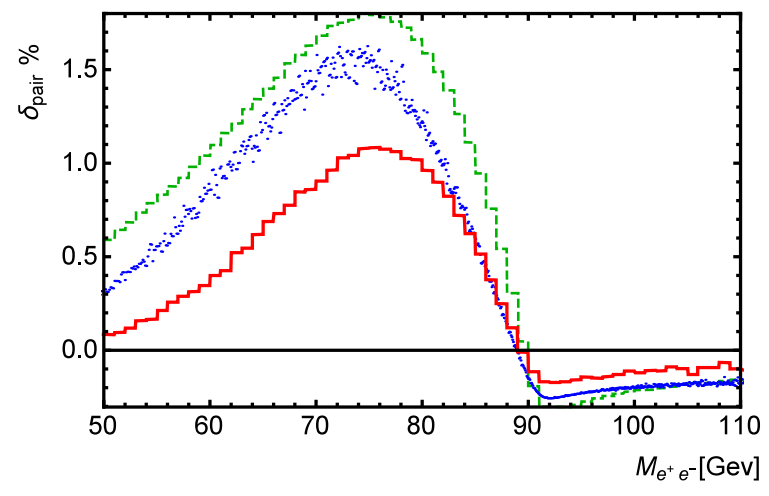

b) Points represent results of simulation by PYTHIA, convoluted bin by bin with formula (26) i.e. as of Ref. [20].

Figure 4: Comparison of PHOTOS and SANC simulations and calculations of extra pair emissions, for the process $p p \rightarrow Z \rightarrow e^{+} e^{-}\left(e^{+} e^{-}\right)$at $14 \mathrm{TeV}$, with independent semi analytical calculations. Correction to lepton pair invariant mass spectrum of PYTHIA generated sample is given in $\%$. Dashed line represents SANC. Solid line represents data by PYTHIA $\times$ PHOTOS. Numerical results obtained with the help of formulae (25) or (26) are superimposed respectively on left and right plot. Our new formula (25) reproduce well results of PHOTOS, but (26) is closer to results of SANC.

- For calculation with formulae (25) 26) we move events, that are generated by PYTHIA, to every possible bin of our test distributions with probabilities obtained from formula (25) or (26) respectively.

- Results from SANC were obtained earlier and we do not recall all details necessary for technical control. They also represent correction for final state emission but spectrum of events prior emission may differ, because slightly different initialization as of Fig. 12 was used. Also, instead of formula (26) equivalent of formula (11) as explained in Section 3 was used. Thus some discrepancy is to be expected.

Analyzing the Fig. 4a, Fig. 5a we can conclude, that PHOTOS is well in agreement with analytical calculation. Numerical precision of agreement is better than $5 \%$ of the pair effect. Estimation is limited by the numerical calculation and CPU time. It can be improved rather easily. The result is supplemented with Fig. 11 of Appendix, which is of more technical nature. It includes plots for muon pair emissions.

If instead, results from formula (26) are used, see Fig. $4 \mathrm{~b}$ and Fig. 5b, results of SANC are much closer than of PHOTOS to that variant of semi-analytical calculation. Taking all these results together we can conclude that we understand numerical difference between PHOTOS and SANC.

The main difference between formula (25) and (26) is that (25) was obtained by rigorous integration over 4-body phase space for final state emissions of matrix element as given 


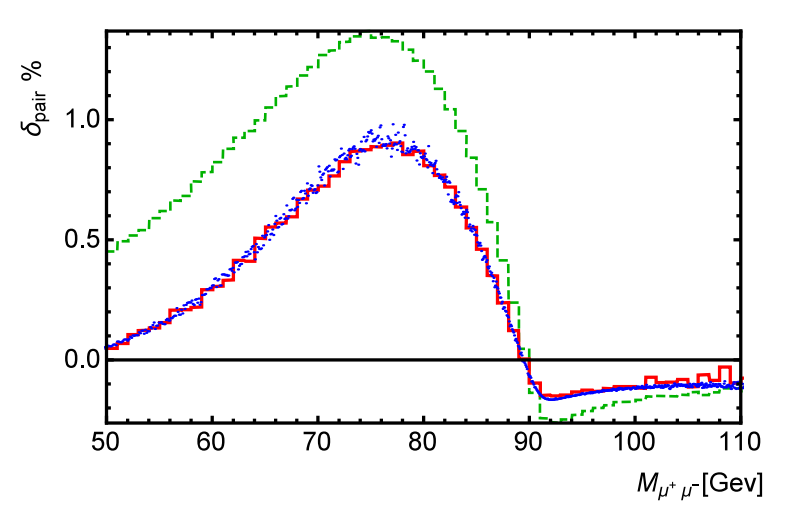

a) Points represent results of simulation by PYTHIA, convoluted bin by bin with our new formula (25).

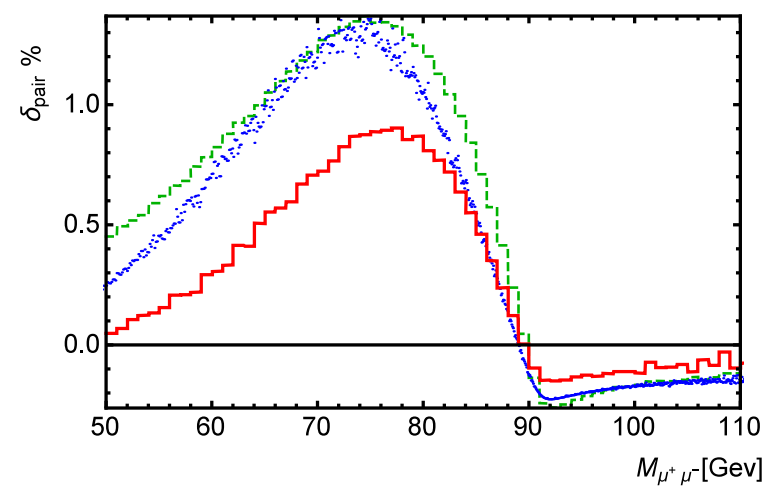

b) Points represent results of simulation by PYTHIA, convoluted bin by bin with formula (26) i.e. as of Ref. [20].

Figure 5: Comparison of PHOTOS and SANC simulations and calculations of extra pair emissions, for the process $p p \rightarrow Z \rightarrow \mu^{+} \mu^{-}\left(e^{+} e^{-}\right)$at $14 \mathrm{TeV}$, with independent semi analytical calculations. Correction to lepton pair invariant mass spectrum of PYTHIA generated sample is given in $\%$. Dashed line represents SANC. Solid line represents data by PYTHIA $\times$ PHOTOS. Numerical results obtained with the help of formulae (25) or (26) are superimposed respectively on left and right plot. Our new formula (25) reproduce well results of PHOTOS, but (26) is closer to results of SANC.

in formula (11). For formula (26) different kinematical conditions (in fact of initial-state emissions) were taken into considerations. If energy of the emitted pair is restricted to soft pair emissions limit, the two calculations coincide, as they should.

One can argue that formula (26) is less suitable for final state pairs emissions. This is not necessarily to be the case. For formula (25) a factorization form of matrix element is used, but such approximation is not used for phase space. This is potential source of numerically important mismatches. Even though exact phase space parametrization offer convenient starting point for future work with matrix element, independent tests with calculations based on four fermions final state matrix elements are of importance.

The PHOTOS can be used as well to analyze an effect of singlet channel, which is the case of misidentification in the detector of first lepton as secondary one, when lepton pair emit lepton pair of the same kind. On Fig. 6. PHOTOS simulations of singlet channel are presented. Number of events fall down logarithmically with rise of invariant mass of misidentified pair. This perfectly agrees with theory.

On Fig. 7, soft pair corrections are presented. The cutoff $\Delta=1 \mathrm{GeV}$ and is applied for energy of the additional lepton pair in the rest frame of colliding partons. This value for cutoff is chosen both to fulfill the conditions $4 m_{f}^{2} \ll \Delta^{2} \ll M_{Z}^{2}$, which correspond to soft pair emissions, and to simulate an effect of the undetected pairs. Depending on the sensitivity of the detector, part of soft lepton pairs remains undetected causing shift in the $p p \rightarrow Z \rightarrow l^{+} l^{-}$spectrum. 


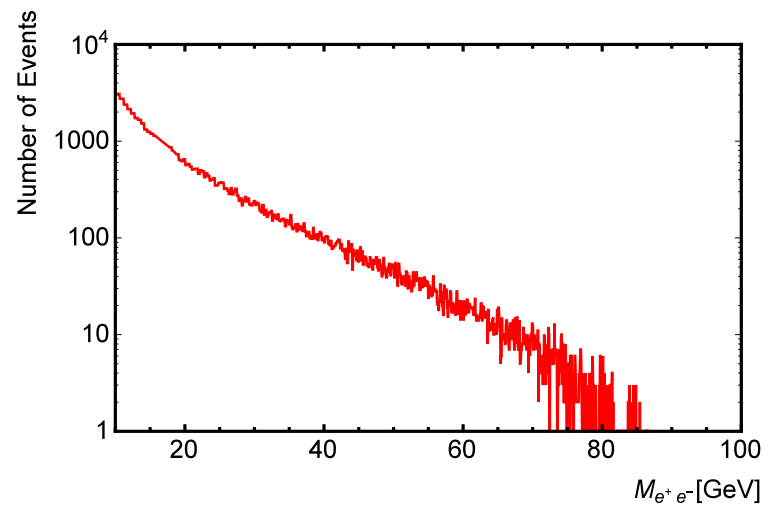

a) $p p \rightarrow Z \rightarrow e^{+} e^{-}\left(e^{+} e^{-}\right)$; probability for presence of additional pair is $\simeq 3 \cdot 10^{-3}$.

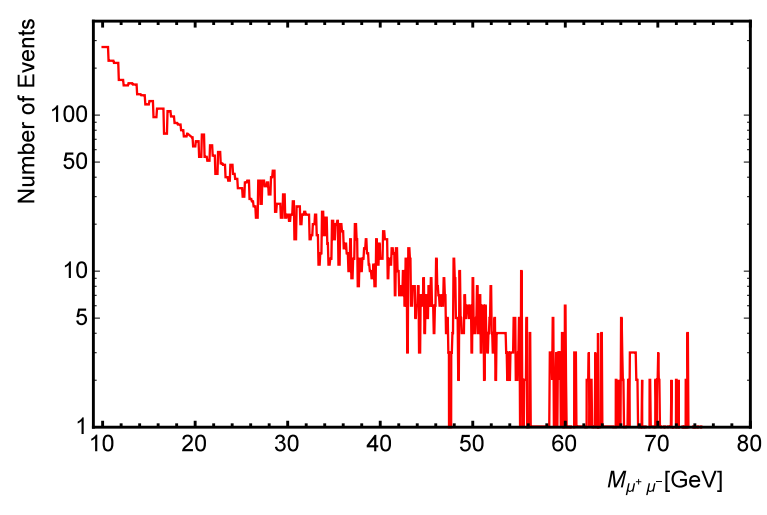

b) $p p \rightarrow Z \rightarrow \mu^{+} \mu^{-}\left(\mu^{+} \mu^{-}\right)$; probability for presence of additional pair is $\simeq 10^{-4}$.

Figure 6: Invariant mass distribution in the singlet channel, i.e. of pair formed from $l^{+}$of emitting pair and $l^{-}$of emitted pair generated by PHOTOS. PYTHIA initialization parameters are presented on Fig. 12, Generated samples (of $\sim 10^{8}$ events), were dominated by configurations with $M\left(l^{+} l^{-}\right) \simeq 10 \mathrm{GeV}$.

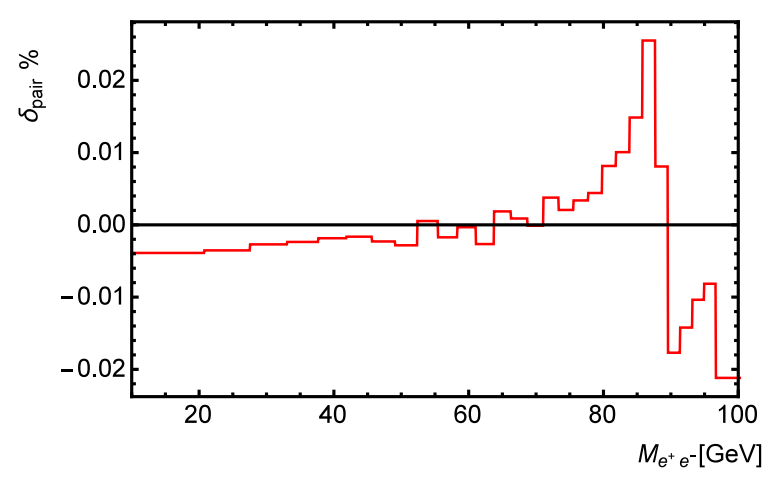

a) $p p \rightarrow Z \rightarrow e^{+} e^{-}\left(e^{+} e^{-}\right)$.

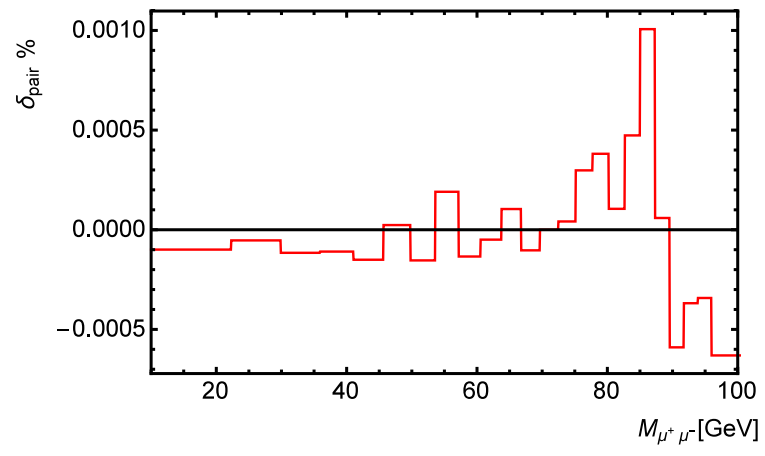

b) $p p \rightarrow Z \rightarrow \mu^{+} \mu^{-}\left(\mu^{+} \mu^{-}\right)$.

Figure 7: Pair correction to spectrum of lepton pair invariant mass of PYTHIA generated sample is given in \%. Original sample is simulated for pp collisions of $14 \mathrm{TeV}$. Solid line represents data by PYTHIA $\times$ PHOTOS. Additional lepton pairs are generated under condition that energy of the additional lepton pair in the rest frame of colliding partons is less than $1 \mathrm{GeV}$. 
The KORALW [21] Monte Carlo can be used to generate $e^{+} e^{-} \rightarrow 4 f$ processes and provide further source of benchmarks for our studies. For that purpose it is necessary to run the program for the Center of Mass Energy equal to $Z$ boson mass and $Z$ width set to a very small value, effectively to switch off emission of pair from initial state. Once parameters of pre-sampler adjusted, program was capable of generating $e^{+} e^{-} \rightarrow Z \rightarrow \mu^{+} \mu^{-} \mu^{+} \mu^{-}$or $e^{+} e^{-} \rightarrow Z \rightarrow \mu^{+} \mu^{-} \tau^{+} \tau^{-}$processes over the full phase space. Once $m_{\tau}$ was replaced with electron mass, all necessary for our testing options were prepared. For PHOTOS sample leptons can originate from emissions or from the pair emitting. In case of $e^{+} e^{-} \mu^{+} \mu^{-}$final state, equal number of $Z \rightarrow e^{+} e^{-}$and $Z \rightarrow \mu^{+} \mu^{-}$decays was used. Normalization for the sample size was fixed to assure $1 \mathrm{M}$ of four-fermion events. Absolute normalization of pair emissions in PHOTOS is verified elsewhere, as explained in Section 2, thanks to tests with analytical formula.

Let us present some numerical results for the samples of $1 \mathrm{M}$ events. In Fig. 8 we present invariant masses of lepton pairs. In Fig. 9 invariant masses for group of three leptons are shown. This is equivalent, for the dominant contribution, to test of the angle between emitted pair and one of the original emitters.

For the muon pair emission in $Z \rightarrow \mu^{+} \mu^{-}$we have prepared only one figure 10, Again, reasonable agreement is shown. Further figures, for all invariant masses which can be constructed from $e^{+} e^{-} \mu^{+} \mu^{-}$or $\mu^{+} \mu^{-} \mu^{+} \mu^{-}$are available from the web page [26].

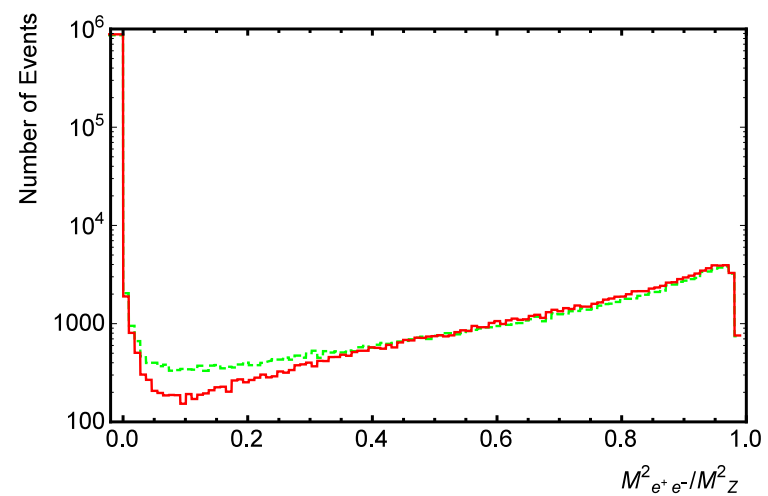

a) Normalized to $M_{Z}^{2}$ spectrum of electron pair mass squared.

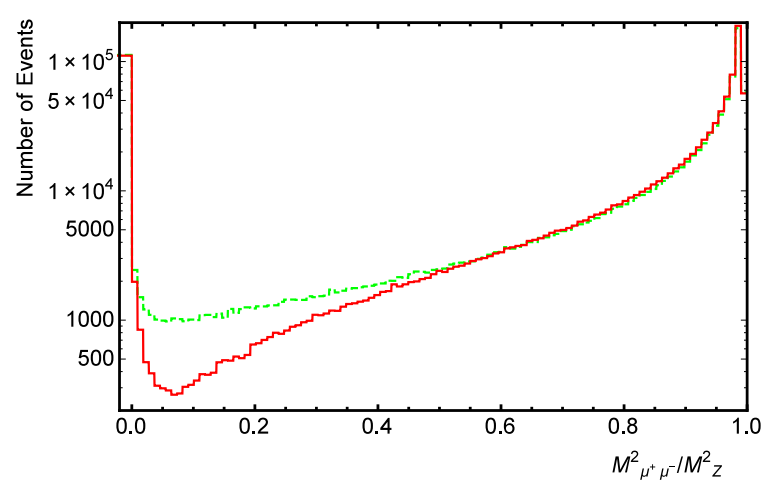

b) Normalized to $M_{Z}^{2}$ spectrum of muon pair mass squared.

Figure 8: Lepton pair invariant mass spectra in the channel $Z \rightarrow \mu^{+} \mu^{-} e^{+} e^{-}$. Results generated by PHOTOS (solid red line) are obtained from samples of equal number of $Z \rightarrow e^{+} e^{-}$and $Z \rightarrow \mu^{+} \mu^{-}$decays. They are compared with results from KORALW (dashed green line) where four fermion final state matrix elements are used as explained in the text. Agreement of most populated bins is of importance for test of PHOTOS.

As expected, in some regions of the phase-space, matrix element based KORALW and pair correction kinematics distribution generated by PHOTOS, vary sizably. This is expected, and of no significance for establishing precision of PHOTOS as generator of pair corrections; the corrections which are themselves at the several permille level only, for the process such 


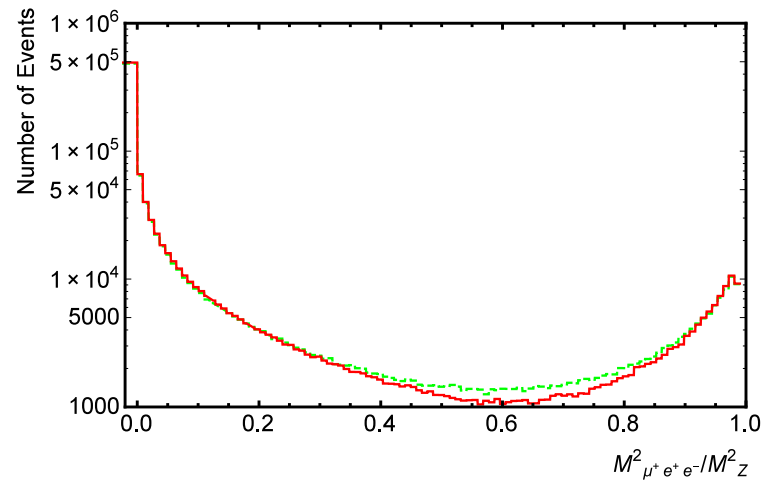

a) Normalized to $M_{Z}^{2}$ spectrum of $\mu^{+} e^{+} e^{-}$ mass squared.

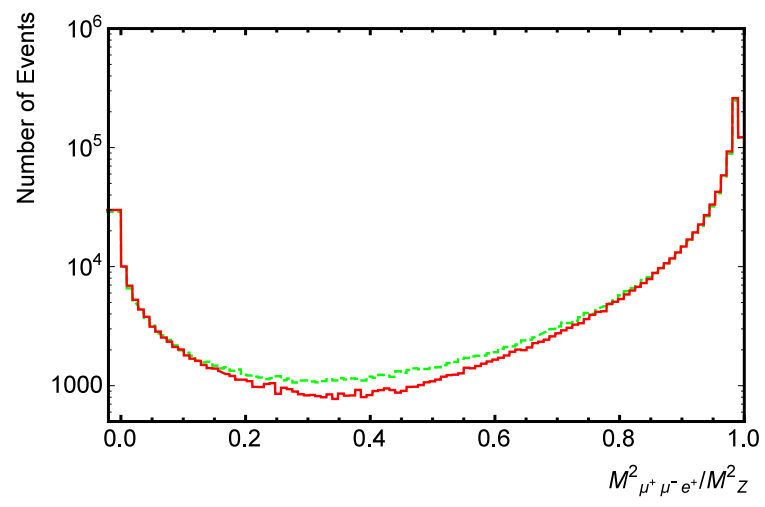

b) Normalized to $M_{Z}^{2}$ spectrum of $\mu^{+} \mu^{-} e^{+}$ mass squared.

Figure 9: Invariant mass spectra in the channel $Z \rightarrow \mu^{+} \mu^{-} e^{+} e^{-}$. Results generated by PHOTOS (solid red line) are obtained from samples of equal number of $Z \rightarrow e^{+} e^{-}$and $Z \rightarrow \mu^{+} \mu^{-}$decays. They are compared with results from KORALW (dashed green line) where four fermion final state matrix elements are used as explained in the text. Agreement of most populated bins is of importance for test of PHOTOS.

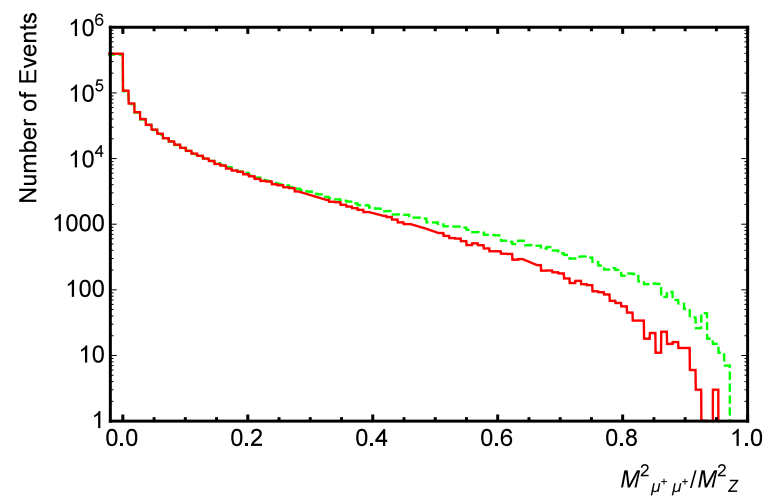

a) Normalized to $M_{Z}^{2}$ spectrum of $\mu^{+} \mu^{-}$ mass squared.

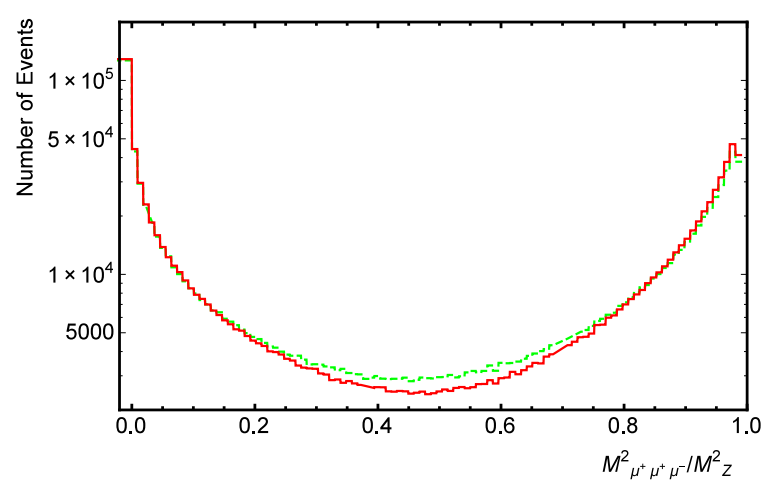

b) Normalized to $M_{Z}^{2}$ spectrum of $\mu^{+} \mu^{+} \mu^{-}$ mass squared.

Figure 10: Invariant mass spectra in the channel $Z \rightarrow \mu^{+} \mu^{-} \mu^{+} \mu^{-}$. Results generated by PHOTOS (solid red line) are obtained from samples of $Z \rightarrow \mu^{+} \mu^{-}$decays. They are compared with results from KORALW (dashed green line) where four fermion final state matrix elements are used as explained in the text. Agreement of most populated bins is of importance for test of PHOTOS. 
as $Z \rightarrow l^{+} l^{-}$decay. For the bins, where bulk of distribution resides, agreement between KORALW and PHOTOS is at the percent level.

Results of the test are encouraging. Good agreement in the region of phase space of soft emissions is obtained. For high energy emissions results from KORALW seem to indicate for somewhat harder spectrum than of PHOTOS, but not as hard as of SANC. This is encouraging observation and clear indication for the future direction of work if higher precision will be needed.

\section{$5 \quad$ Higher order effects}

Both SANC and PHOTOS can generate pair effects simultaneously with emission of photons. Because of rather steep energy spectrum for emitted pairs, the effect of photonic bremsstrahlung on pair emission is not expected to be large. To validate this expectation we have introduced the following option into PHOTOS; instead of generating in $50 \%$ of cases, pair emission before algorithm for photon emission is involved we have always generated pairs as the last step. Standard tests with the help of MC-TESTER demonstrate about $4 \%$ increase in the number of final states consisting of configurations with added pair and at least one real photon of energy above $1 \mathrm{GeV}$. Shapes of distributions remained not modified in a noticeable way for the sample of $100 \mathrm{MeV}$ events (see [26]).

This provides not only consistency check, but also confirms that PHOTOS can be used with generator such as KKMC [27] for generation of final state pair emissions. This, of course, require that intermediate $Z / \gamma^{*}$ state is present in the event record. Such intermediate state can be obtained from the low level generation of KKMC. Even if it is not physically justified to define $Z / \gamma^{*}$ intermediate state once initial-final state interference is taken into account, resulting inconsistency is only at the \% level, at most, of the pair emission effect which itself is at $\%$ level too. It is thus at the $10^{-4}$ precision level.

\section{Conclusions}

We can conclude that we control bulk of pair effects, down to $10 \%$ of their size in the regions of phase space of importance for experimental conditions, that is for emitted pairs of rather small energies, or collinear. Rare events featuring hard pairs, could bring larger ambiguities, but are expected also to be outside of experimental acceptance. For this region of phase space taken separately, uncertainty is larger, of order of even $50 \%$, but on the other hand, events of such configurations contribute to the overall Drell-Yan sample at sub-permille level.

The origin of the differences between PHOTOS and SANC results used for the systematic error evaluation is localized and confirmed with semi-analytical calculation. It is due to approximation resulting from how eq. (11) is used in PHOTOS and in SANC. Phase space, as used in PHOTOS algorithm, is explicit and exact, enabling for straightforward improvement of matrix element. Note that PHOTOS usage of approximation in matrix element, but not 
in phase space, may not be optimal. This is why solution used in SANC, a priori, is not of lower precision than that of PHOTOS. We argue to improve the precision tag from $0.3 \%$ to $0.1 \%$ for the pair implementation of the two programs and in applications for observables relevant for heavy boson reconstruction. We provide indications for steps necessary to improve beyond $0.1 \%$ precision level.

For the estimation of ambiguities size, the comparisons with KORALW, where complete $2 \rightarrow 4$ fermion matrix element is available, was instrumental. It may need to be continued in the future, but as hard pairs contribute to the bulk of differences, it may not be of urgency for present day experimental effort. This region of phase-space is expected to remain outside of experimental acceptance.

\section{Acknowledgments}

R.S. is grateful for a financial support from ENIGMAS program and to kind hospitality of the ATLAS group in LAPP. This work was partially supported by the funds of Polish National Science Center under decision UMO-2014/15/B/ST2/00049. Useful discussions with Maciej Skrzypek and also his help with KORALW installation are appreciated. The work is supported in part by the Programme of the French-Polish Cooperation between IN2P3 and COPIN within the collaborations Nos. 10-138 and 11-142.

\section{References}

[1] A. Arbuzov, R. Sadykov, and Z. Was, Eur.Phys.J. C73 (2013), no. 11 2625, 1212.6783 .

[2] E. Barberio, B. van Eijk, and Z. Was, Comput.Phys.Commun. 66 (1991) 115-128.

[3] E. Barberio and Z. Was, Comput.Phys.Commun. 79 (1994) 291-308.

[4] P. Golonka and Z. Was, Eur.Phys.J. C45 (2006) 97-107, hep-ph/0506026.

[5] G. Nanava and Z. Was, Eur.Phys.J. C51 (2007) 569-583, hep-ph/0607019.

[6] P. Golonka and Z. Was, Eur.Phys.J. C50 (2007) 53-62, hep-ph/0604232.

[7] G. Nanava, Q. Xu, and Z. Was, Eur.Phys.J. C70 (2010) 673-688, 0906.4052.

[8] N. Davidson, T. Przedzinski, and Z. Was, Comput. Phys. Commun. 199 (2016) 86$101,1011.0937$.

[9] A. Andonov, A. Arbuzov, D. Bardin, S. Bondarenko, P. Christova, et al., Comput.Phys.Commun. 174 (2006) 481-517, hep-ph/0411186.

[10] A. Arbuzov, D. Bardin, S. Bondarenko, P. Christova, L. Kalinovskaya, et al., Eur.Phys.J. C46 (2006) 407-412, hep-ph/0506110. 
[11] A. Arbuzov, D. Bardin, S. Bondarenko, P. Christova, L. Kalinovskaya, et al., Eur.Phys.J. C54 (2008) 451-460, 0711.0625.

[12] A. Arbuzov and R. Sadykov, J.Exp.Theor.Phys. 106 (2008) 488-494, 0707.0423.

[13] A. Andonov, A. Arbuzov, S. Bondarenko, P. Christova, V. Kolesnikov, et al., Phys.Part.Nucl.Lett. 4 (2007) 451-460.

[14] A. Andonov, A. Arbuzov, D. Bardin, S. Bondarenko, P. Christova, et al., Comput.Phys.Commun. 181 (2010) 305-312, 0812.4207.

[15] A. Andonov, A. Arbuzov, S. Bondarenko, P. Christova, V. Kolesnikov, et al., Phys.Atom.Nucl. 73 (2010) 1761-1769, 0901.2785.

[16] D. Bardin, S. Bondarenko, P. Christova, L. Kalinovskaya, L. Rumyantsev, et al., JETP Lett. 96 (2012) 285-289, 1207.4400.

[17] A. Arbuzov, Phys.Lett. B470 (1999) 252-258, hep-ph/9908361.

[18] A. Arbuzov, JHEP 0107 (2001) 043.

[19] N. Davidson, P. Golonka, T. Przedzinski, and Z. Was, Comput.Phys.Commun. 182 (2011) 779-789, 0812.3215.

[20] S. Jadach, M. Skrzypek, and B. Ward, Phys.Rev. D49 (1994) 1178-1182.

[21] S. Jadach, W. Placzek, M. Skrzypek, B. Ward, and Z. Was, Comput.Phys.Commun. 119 (1999) 272-311, hep-ph/9906277.

[22] C. M. Carloni Calame, G. Montagna, O. Nicrosini, and M. Treccani, Phys. Rev. D69 (2004) 037301, hep-ph/0303102.

[23] S. Alioli et al., Eur. Phys. J. C77 (2017), no. 11 280, 1606.02330.

[24] S. Antropov, "Effect of emission of extra lepton pair for precise measurement of Wboson mass", in 23rd Cracow Epiphany Conference on Particle Theory Meets the First Data from LHC Run 2 Cracow, Poland, January 9-12, 2017, 2017, 1704.06203.

[25] T. Sjostrand, S. Mrenna, and P. Z. Skands, Comput. Phys. Commun. 178 (2008) $852-867,0710.3820$.

[26] Further numerical results are added here, http://annapurna.ifj.edu.pl/ wasm/phNLO.htm.

[27] S. Jadach, B. Ward, and Z. Was, Comput.Phys.Commun. 130 (2000) 260-325, hep-ph/9912214.

[28] J. C. Ward, Phys. Rev. 78 (1950) 182.

[29] Z. Was, "Radiative corrections", in 1993 European School of High-Energy Physics, Zakopane, Poland, 12-25 Sep 1993: Proceedings, pp. 307-338, 1994. 


\section{A Appendix.}

Let us collect formulae of our calculation used to understand details of analytic calculation of ref. [20]. We have prepared variant of analytic calculation matching solution used in PHOTOS. We start from the phase-space parametrization and integration of matrix element follows.

\section{A.1 Parametrization of the phase space.}

$$
\begin{aligned}
& \Omega=\quad \int \frac{d^{3} q_{1}}{2\left(q_{1}\right)_{0}(2 \pi)^{3}} \cdot \frac{d^{3} q_{2}}{2\left(q_{2}\right)_{0}(2 \pi)^{3}} \cdot \frac{d^{3} p}{2 p_{0}(2 \pi)^{3}} \cdot \frac{d^{3} p^{\prime}}{2 p_{0}^{\prime}(2 \pi)^{3}}(2 \pi)^{4} \delta^{4}\left(R-p-p^{\prime}-q_{1}-q_{2}\right)= \\
& =\int d^{4} q d^{4} Q \frac{d^{3} q_{1}}{2\left(q_{1}\right)_{0}(2 \pi)^{3}} \cdot \frac{d^{3} q_{2}}{2\left(q_{2}\right)_{0}(2 \pi)^{3}} \cdot \frac{d^{3} p}{2 p_{0}(2 \pi)^{3}} \cdot \frac{d^{3} p^{\prime}}{2 p_{0}^{\prime}(2 \pi)^{3}}(2 \pi)^{4} \times \\
& \times \quad \delta^{4}\left(R-p-p^{\prime}-q_{1}-q_{2}\right) \delta^{4}\left(q-q_{1}-q_{2}\right) \delta^{4}\left(Q-p-p^{\prime}\right) \\
& \int \frac{d^{3} q_{1}}{2\left(q_{1}\right)^{0}} \frac{d^{3} q_{2}}{2\left(q_{2}\right)^{0}} \delta^{4}\left(q-q_{1}-q_{2}\right)=\int \frac{\left|\overline{q_{1}}\right| d \cos \theta_{q_{1}} d \phi_{q_{1}}}{4 \sqrt{q^{2}}},
\end{aligned}
$$

where $\theta_{q_{1}}, \phi_{q_{1}}$ are direction of $q_{1}$ in the rest frame of $q,\left|\overline{q_{1}}\right|=\left|\overline{q_{2}}\right|=\sqrt{\frac{q^{2}}{4}-\mu^{2}}$.

$$
\int \frac{d^{3} p}{2(p)^{0}} \frac{d^{3} p^{\prime}}{2\left(p^{\prime}\right)^{0}} \delta^{4}\left(Q-p-p^{\prime}\right)=\int \frac{|\bar{p}| d \cos \theta_{p} d \phi_{p}}{4 \sqrt{p^{2}}}
$$

where $\theta_{p}, \phi_{p}$ are direction of $p$ in the rest frame of $Q,|\bar{p}|=\left|\overline{p^{\prime}}\right|=\sqrt{\frac{Q^{2}}{4}-m^{2}}$.

$$
\int d^{4} q d^{4} Q \delta^{4}(R-Q-q)=\int\left(d \cos \theta_{q} d \phi_{q}\right) d M_{Q}^{2} d M_{q}^{2} \frac{\sqrt{\lambda}}{8 s}
$$

where $\theta_{q}, \phi_{q}$ are direction of $q$ in the rest frame of $R$.

$$
\Omega=\frac{1}{(2 \pi)^{8}} \int d M_{q}^{2} d M_{Q}^{2} d \cos \theta_{q_{1}} d \phi_{q_{1}} d \cos \theta_{p} d \phi_{p} d \cos \theta_{q} d \phi_{q} \frac{1}{8} \sqrt{1-\frac{4 \mu^{2}}{q^{2}}} \frac{1}{8} \sqrt{1-\frac{4 m^{2}}{Q^{2}}} \frac{\sqrt{\lambda\left(s, M_{Q}^{2}, M_{q}^{2}\right)}}{8 s} .
$$

We choose that:

1. $\theta_{p}, \phi_{p}$ define orientation of $p$ (in the rest frame of $Q$ ) with respect to $z$ axis along direction of $\mathrm{q}$ (as seen in this frame);

2. $\theta_{q_{1}}, \phi_{q_{1}}$ define orientation of $q_{1}$ (in the rest frame of $q$ ) with respect to $z$ axis along boost from this frame to the rest frame of $Q$;

3. $\theta_{q}, \phi_{q}$ define orientation of $p$ with respect to laboratory directions (in the rest frame of $R$ ). 


\section{A.2 Preparation of the Matrix Element.}

Let us now turn our attention to matrix element. Factorized term obtained from pair emission matrix element and used in ref. [20] formula (1) as integrand reads:

$$
F\left(p, p^{\prime}, q, q_{1}, q_{2}, a\right)=\left(\frac{\alpha}{\pi}\right)^{2} \frac{1}{\pi^{2}}\left(\frac{2 p-a q}{a q^{2}-2 p q}-\frac{2 p^{\prime}-a q}{a q^{2}-2 p^{\prime} q}\right)_{\mu}\left(\frac{2 p-a q}{a q^{2}-2 p q}-\frac{2 p^{\prime}-a q}{a q^{2}-2 p^{\prime} q}\right)_{\nu} \frac{4 q_{1}^{\mu} q_{2}^{\nu}-q^{2} g^{\mu \nu}}{2 q^{4}} .
$$

Note that it includes factor $\frac{1}{(2 \pi)^{6}}$ of the phase-space integration volume. We need to recall that at the end of calculation.

Now we can express all four vectors necessary for formula (7) with the help of previously specified angles. Four vectors $p, p^{\prime}, q, q_{1}, q_{2}$ in the rest frame of $Q$ read:

$$
\begin{aligned}
p & =\left(E_{p}, p \cos \phi_{p} \sin \theta_{p}, p \sin \phi_{p} \sin \theta_{p}, p \cos \theta_{p}\right) \\
p^{\prime} & =\left(E_{p},-p \cos \phi_{p} \sin \theta_{p},-p \sin \phi_{p} \sin \theta_{p},-p \cos \theta_{p}\right), \\
q & =\left(E_{q}, 0,0, q\right)
\end{aligned}
$$

where

$$
\begin{aligned}
E_{p} & =\frac{1}{2} M_{Q}, \\
p & =\sqrt{\frac{M_{Q}^{2}}{4}-m^{2},} \\
E_{q} & =\frac{s-M_{Q}^{2}-M_{q}^{2}}{2 M_{Q}}, \\
q & =\frac{\sqrt{\left(s-M_{Q}^{2}-M_{q}^{2}\right)^{2}-4 M_{Q}^{2} M_{q}^{2}}}{2 M_{Q}} .
\end{aligned}
$$

To obtain expressions for $E_{q}$ and $q$ formulae for $p$ and $p^{\prime}$ and $s=\left(p+p^{\prime}+q\right)^{2}$ are needed.

We first define $q_{1}$ and $q_{2}$ in the the rest frame of $q$ :

$$
\begin{aligned}
& q_{1}=\left(\frac{M_{q}}{2}, v \cos \phi_{q_{1}} \sin \theta_{q_{1}}, v \sin \phi_{q_{1}} \sin \theta_{q_{1}}, v \cos \theta_{q_{1}}\right), \\
& q_{2}=\left(\frac{M_{q}}{2},-v \cos \phi_{q_{1}} \sin \theta_{q_{1}},-v \sin \phi_{q_{1}} \sin \theta_{q_{1}},-v \cos \theta_{q_{1}}\right),
\end{aligned}
$$

where

$$
v=\sqrt{\frac{M_{q}^{2}}{4}-\mu^{2}} .
$$

\section{A.3 Integration of matrix element.}

We have to calculate

$$
\sigma=\int d \Omega F\left|M_{B}\right|^{2}
$$


where $F$ if given by formula (7) and $d \Omega$ by (6).$\left|M_{B}\right|^{2}$ is not important as we will see.

Question is how to do it in most convenient way without loosing symmetry properties of (7).

Observation:

1. $F$ depends on all variables except $\theta_{q}, \phi_{q}$;

2. $\left|M_{B}\right|^{2}$ depends only on $\theta_{q}, \phi_{q}$;

3. $\theta_{q_{1}}, \phi_{q_{1}}$ are present only in $\frac{4 q_{1}^{\mu} q_{2}^{\nu}-q^{2} g^{\mu \nu}}{2 q^{4}}$.

It is convenient to integrate $\frac{4 q_{1}^{\mu} q_{2}^{\nu}-q^{2} g^{\mu \nu}}{2 q^{4}}$ over $\theta_{q_{1}}, \phi_{q_{1}}$ in the rest frame of $q$. Because of Lorentz invariance we have

$$
\int d \theta_{q} d \phi_{q} d \frac{4 q_{1}^{\mu} q_{2}^{\nu}-q^{2} g^{\mu \nu}}{2 q^{4}}=X g^{\mu \nu}+Y q^{\mu} q^{\nu}
$$

Thus

$$
\begin{aligned}
& \int d \theta_{q} d \phi_{q} d \frac{4 q_{1}^{\mu} q_{2}^{\nu}-q^{2} g^{\mu \nu}}{2 q^{4}}= \\
& =\frac{16 \pi}{2 M_{q}^{4}}\left(\begin{array}{cccc}
\frac{M_{q}^{2}}{4} & 0 & 0 & 0 \\
0 & -\frac{1}{3}\left(\frac{M_{q}^{2}}{4}-\mu^{2}\right) & 0 & 0 \\
0 & 0 & -\frac{1}{3}\left(\frac{M_{q}^{2}}{4}-\mu^{2}\right) & 0 \\
0 & 0 & 0 & -\frac{1}{3}\left(\frac{M_{q}^{2}}{4}-\mu^{2}\right)
\end{array}\right)-\frac{4 \pi M_{q}^{2}}{2 M_{q}^{4}}\left(\begin{array}{cccc}
1 & 0 & 0 & 0 \\
0 & -1 & 0 & 0 \\
0 & 0 & -1 & 0 \\
0 & 0 & 0 & -1
\end{array}\right)= \\
& =\frac{1}{M_{q}^{2}}\left(\begin{array}{cccc}
0 & 0 & 0 & 0 \\
0 & \frac{4 \pi}{3}\left(1+\frac{2 \mu^{2}}{M_{q}^{2}}\right) & 0 & 0 \\
0 & 0 & \frac{4 \pi}{3}\left(1+\frac{2 \mu^{2}}{M_{q}^{2}}\right) & 0 \\
0 & 0 & 0 & \frac{4 \pi}{3}\left(1+\frac{2 \mu^{2}}{M_{q}^{2}}\right)
\end{array}\right)= \\
& =-\frac{1}{M_{q}^{2}} \cdot \frac{4 \pi}{3}\left(1+\frac{2 \mu^{2}}{M_{q}^{2}}\right)\left(\begin{array}{cccc}
1 & 0 & 0 & 0 \\
0 & -1 & 0 & 0 \\
0 & 0 & -1 & 0 \\
0 & 0 & 0 & -1
\end{array}\right)+\frac{1}{M_{q}^{2}} \cdot \frac{4 \pi}{3}\left(1+\frac{2 \mu^{2}}{M_{q}^{2}}\right)\left(\begin{array}{cccc}
1 & 0 & 0 & 0 \\
0 & 0 & 0 & 0 \\
0 & 0 & 0 & 0 \\
0 & 0 & 0 & 0
\end{array}\right)= \\
& =-\frac{1}{M_{q}^{2}} \cdot \frac{4 \pi}{3}\left(1+\frac{2 \mu^{2}}{M_{q}^{2}}\right) g^{\mu \nu}+\frac{1}{M_{q}^{2}} \cdot \frac{4 \pi}{3}\left(1+\frac{2 \mu^{2}}{M_{q}^{2}}\right) \frac{q^{\mu} q^{\nu}}{M_{q}^{2}} .
\end{aligned}
$$

It is easy to verify, that

$$
\left(\frac{2 p-a q}{a q^{2}-2 p q}-\frac{2 p^{\prime}-a q}{a q^{2}-2 p^{\prime} q}\right)_{\mu}\left(\frac{2 p-a q}{a q^{2}-2 p q}-\frac{2 p^{\prime}-a q}{a q^{2}-2 p^{\prime} q}\right)_{\nu} q^{\mu} q^{\nu}
$$

equals zero, and second part of (13) does not contribute. This is a consequence of property resulting from Ward identity of QED [28]. 
Products of four-vectors can be expressed with the help of invariants and masses used in phase-space parametrization

$$
\begin{aligned}
p \cdot p^{\prime} & =\frac{M_{Q}^{2}}{2}-m^{2} ; \\
p \cdot q & =\frac{s-M_{Q}^{2}-M_{q}^{2}}{4}-\sqrt{\frac{M_{Q}^{2}}{4}-m^{2}} \frac{\lambda^{\frac{1}{2}}\left(s, M_{Q}^{2}, M_{q}^{2}\right)}{2 M_{Q}} \cos \theta_{p} ; \\
p^{\prime} \cdot q & =\frac{s-M_{Q}^{2}-M_{q}^{2}}{4}+\sqrt{\frac{M_{Q}^{2}}{4}-m^{2}} \frac{\lambda^{\frac{1}{2}}\left(s, M_{Q}^{2}, M_{q}^{2}\right)}{2 M_{Q}} \cos \theta_{p} .
\end{aligned}
$$

In case of $a=0$ calculation is particularly simple:

$$
\begin{aligned}
& \left(\frac{2 p-a q}{a q^{2}-2 p q}-\frac{2 p^{\prime}-a q}{a q^{2}-2 p^{\prime} q}\right)^{2}= \\
= & \frac{4 m^{2}}{\left(\frac{s-M_{Q}^{2}-M_{q}^{2}}{2}-\sqrt{\frac{M_{Q}^{2}}{4}-m^{2}} \frac{\lambda^{\frac{1}{2}}\left(s, M_{Q}^{2}, M_{q}^{2}\right)}{M_{Q}} \cos \theta_{p}\right)^{2}}+ \\
+ & \frac{4 m^{2}}{\left(\frac{s-M_{Q}^{2}-M_{q}^{2}}{2}+\sqrt{\frac{M_{Q}^{2}}{4}-m^{2}} \frac{\lambda^{\frac{1}{2}}\left(s, M_{Q}^{2}, M_{q}^{2}\right)}{M_{Q}} \cos \theta_{p}\right)^{2}}- \\
- & 2 \frac{2 M_{Q}^{2}-4 m^{2}}{\frac{\left(s-M_{Q}^{2}-M_{q}^{2}\right)^{2}}{4}-\left(\frac{M_{Q}^{2}}{4}-m^{2}\right) \frac{\lambda\left(s, M_{Q}^{2}, M_{q}^{2}\right)}{M_{Q}^{2}} \cos ^{2} \theta_{p}} .
\end{aligned}
$$

In general case thanks to (8) we obtain

$$
\begin{aligned}
& \left(\frac{2 p-a q}{a q^{2}-2 p q}-\frac{2 p^{\prime}-a q}{a q^{2}-2 p^{\prime} q}\right)^{2}=\left(\frac{4 p^{\mu} p_{\mu}+a^{2} q^{\mu} q_{\mu}-4 a p_{\mu} q^{\mu}}{\left(a q^{\mu} q_{\mu}-2 E_{p} E_{q}+2 p q \cos \theta_{p}\right)^{2}}+\frac{4 p^{\mu} p_{\mu}+a^{2} q^{\mu} q_{\mu}-4 a p_{\mu}^{\prime} q^{\mu}}{\left(a q^{\mu} q_{\mu}-2 E_{p} E_{q}-2 p q \cos \theta_{p}\right)^{2}}\right. \\
& \left.-2 \frac{4 p^{\mu} p_{\mu}^{\prime}-2 a q_{\mu}\left(p+p^{\prime}\right)^{\mu}+a^{2} q^{\mu} q_{\mu}}{\left(a q^{\mu} q_{\mu}-2 E_{p} E_{q}+2 p q \cos \theta_{p}\right)\left(a q^{\mu} q_{\mu}-2 E_{p} E_{q}-2 p q \cos \theta_{p}\right)}\right) \\
& =\left(\frac{4 m^{2}+a M_{q}^{2}-4 a E_{p} E_{q}+4 a p q \cos \theta_{p}}{\left(a M_{q}^{2}-2 E_{p} E_{q}+2 p q \cos \theta_{p}\right)^{2}}\right. \\
& \left.+\frac{4 m^{2}+a M_{q}^{2}-4 a E_{p} E_{q}-4 a p q \cos \theta_{p}}{\left(a M_{q}^{2}-2 E_{p} E_{q}-2 p q \cos \theta_{p}\right)^{2}}-2 \frac{4\left(m^{2}+2 p^{2}\right)-4 a E_{q} E_{p}+a^{2} M_{q}^{2}}{\left(a M_{q}^{2}-2 E_{p} E_{q}\right)^{2}-4 p^{2} q^{2} \cos ^{2} \theta_{p}}\right) .
\end{aligned}
$$

In order to integrate expression (17) over $\cos \theta_{p}$ we separate it into three parts, corresponding to distinct polynomials in $\cos \theta_{p}$. Integrals read: 


$$
\begin{aligned}
C_{1} & =\int_{1}^{-1} d \cos \theta_{p}\left(\frac{4 m^{2}+a M_{q}^{2}-4 a E_{p} E_{q}}{\left(a M_{q}^{2}-2 E_{p} E_{q}+2 p q \cos \theta_{p}\right)^{2}}+\frac{4 m^{2}+a M_{q}^{2}-4 a E_{p} E_{q}}{\left(a M_{q}^{2}-2 E_{p} E_{q}-2 p q \cos \theta_{p}\right)^{2}}\right) \\
C_{2} & =\int_{1}^{-1} d \cos \theta_{p}\left(\frac{4 a p q \cos \theta_{p}}{\left(a M_{q}^{2}-2 E_{p} E_{q}+2 p q \cos \theta_{p}\right)^{2}}-\frac{4 a p q \cos \theta_{p}}{\left(a M_{q}^{2}-2 E_{p} E_{q}-2 p q \cos \theta_{p}\right)^{2}}\right) \\
C_{3} & =\int_{1}^{-1} d \cos \theta_{p} \frac{4\left(m^{2}+2 p^{2}\right)-4 a E_{q} E_{p}+a^{2} M_{q}^{2}}{\left(a M_{q}^{2}-2 E_{p} E_{q}\right)^{2}-4 p^{2} q^{2} \cos ^{2} \theta_{p}} .
\end{aligned}
$$

Let us now return to our main eq. (11). We get

$$
\begin{aligned}
\sigma & =\frac{1}{(2 \pi)^{8}} \frac{1}{\pi^{2}} \int\left|M_{B}\right|^{2} d M_{q}^{2} d M_{Q}^{2} d \cos \theta_{p} d \phi_{p} d \cos \theta_{q} d \phi_{q} \frac{1}{8} \sqrt{1-\frac{4 \mu^{2}}{q^{2}}} \frac{1}{8} \sqrt{1-\frac{4 m^{2}}{Q^{2}}} \frac{\sqrt{\lambda\left(s, M_{Q}^{2}, M_{q}^{2}\right)}}{8 s} \times \\
& \times\left(\frac{\alpha}{\pi}\right)^{2}\left(\frac{2 p-a q}{a q^{2}-2 p q}-\frac{2 p^{\prime}-a q}{a q^{2}-2 p^{\prime} q}\right)_{\mu}\left(\frac{2 p-a q}{a q^{2}-2 p q}-\frac{2 p^{\prime}-a q}{a q^{2}-2 p^{\prime} q}\right)^{\mu} \frac{1}{M_{q}^{2}} \cdot \frac{(-4 \pi)}{3}\left(1+\frac{2 \mu^{2}}{M_{q}^{2}}\right)
\end{aligned}
$$

or after re-ordering of terms

$$
\begin{aligned}
\sigma & =-\frac{1}{3 \cdot 2^{15} \pi^{9} s}\left(\frac{\alpha}{\pi}\right)^{2} \int\left[\left|M_{B}\right|^{2} d \cos \theta_{q} d \phi_{q}\right] d M_{Q}^{2} \frac{d M_{q}^{2}}{M_{q}^{2}} d \cos \theta_{p} d \phi_{p} \sqrt{1-\frac{4 \mu^{2}}{M_{q}^{2}}}\left(1+\frac{2 \mu^{2}}{M_{q}^{2}}\right) \sqrt{1-\frac{4 m^{2}}{M_{Q}^{2}}} \times \\
& \times \lambda^{\frac{1}{2}}\left(s, M_{Q}^{2}, M_{q}^{2}\right)\left(\frac{2 p-a q}{a q^{2}-2 p q}-\frac{2 p^{\prime}-a q}{a q^{2}-2 p^{\prime} q}\right)_{\mu}\left(\frac{2 p-a q}{a q^{2}-2 p q}-\frac{2 p^{\prime}-a q}{a q^{2}-2 p^{\prime} q}\right)^{\mu} .
\end{aligned}
$$

We simplify integral (20) with the help of (16). Expression (15) or(17) does not depend on $\phi_{p}$, integration over $\phi_{p}$ is trivial and gives an overall factor $2 \pi$. One also notice that integrals over $\cos \theta_{p}$ of first and second part of (16) are equal. We obtain

$$
\begin{aligned}
\sigma & =-\frac{1}{3 \cdot 2^{15} \pi^{9} s}\left(\frac{\alpha}{\pi}\right)^{2} \int\left[\left|M_{B}\right|^{2} d \cos \theta_{q} d \phi_{q}\right] d M_{Q}^{2} \frac{d M_{q}^{2}}{M_{q}^{2}} 2 \pi \sqrt{1-\frac{4 \mu^{2}}{M_{q}^{2}}}\left(1+\frac{2 \mu^{2}}{M_{q}^{2}}\right) \sqrt{1-\frac{4 m^{2}}{M_{Q}^{2}}} \times \\
& \times \lambda^{\frac{1}{2}}\left(s, M_{Q}^{2}, M_{q}^{2}\right) \int_{1}^{-1} d \cos \theta_{p}\left[\frac{8 m^{2}}{\left(\frac{s-M_{Q}^{2}-M_{q}^{2}}{2}-\sqrt{\frac{M_{Q}^{2}}{4}-m^{2}} \frac{\lambda^{\frac{1}{2}}\left(s, M_{Q}^{2}, M_{q}^{2}\right)}{M_{Q}} \cos \theta_{p}\right)^{2}}-\right. \\
& \left.-2 \frac{2 M_{Q}^{2}-4 m^{2}}{\frac{\left(s-M_{Q}^{2}-M_{q}^{2}\right)^{2}}{4}-\left(\frac{M_{Q}^{2}}{4}-m^{2}\right) \frac{\lambda\left(s, M_{Q}^{2}, M_{q}^{2}\right)}{M_{Q}^{2}} \cos ^{2} \theta_{p}}\right] .
\end{aligned}
$$

Now we need to integrate over $\cos \theta_{p}$. The following formulas are helpful

$$
\int_{-1}^{1} \frac{d x}{(A-B x)^{2}}=\frac{2}{A^{2}-B^{2}}
$$


and

$$
\int_{-1}^{1} \frac{d x}{A^{2}-B^{2} x^{2}}=-\frac{1}{A B} \ln \frac{A-B}{A+B} .
$$

With help of these, we get:

$$
\begin{aligned}
& \sigma=-\frac{1}{3 \cdot 2^{15} \pi^{9} s}\left(\frac{\alpha}{\pi}\right)^{2} \int\left[\left|M_{B}\right|^{2} d \cos \theta_{q} d \phi_{q}\right] d M_{Q}^{2} \frac{d M_{q}^{2}}{M_{q}^{2}} 2 \pi \sqrt{1-\frac{4 \mu^{2}}{M_{q}^{2}}}\left(1+\frac{2 \mu^{2}}{M_{q}^{2}}\right) \sqrt{1-\frac{4 m^{2}}{M_{Q}^{2}}} \times \\
& \times \quad \lambda^{\frac{1}{2}}\left(s, M_{Q}^{2}, M_{q}^{2}\right)\left[\frac{16 m^{2}}{\frac{\left(s-M_{Q}^{2}-M_{q}^{2}\right)^{2}}{4}-\left(\frac{M_{Q}^{2}}{4}-m^{2}\right) \frac{\lambda\left(s, M_{Q}^{2}, M_{q}^{2}\right)}{M_{Q}^{2}}}+\right. \\
& \left.+2 \frac{2 M_{Q}^{2}-4 m^{2}}{\frac{s-M_{Q}^{2}-M_{q}^{2}}{2} \sqrt{\frac{M_{Q}^{2}}{4}-m^{2}} \frac{\lambda^{\frac{1}{2}}\left(s, M_{Q}^{2}, M_{q}^{2}\right)}{M_{Q}}} \ln \frac{\frac{s-M_{Q}^{2}-M_{q}^{2}}{2}-\sqrt{\frac{M_{Q}^{2}}{4}-m^{2}} \frac{\lambda^{\frac{1}{2}}\left(s, M_{Q}^{2}, M_{q}^{2}\right)}{M_{Q}}}{\frac{s-M_{Q}^{2}-M_{q}^{2}}{2}+\sqrt{\frac{M_{Q}^{2}}{4}-m^{2}} \frac{\lambda^{\frac{1}{2}}\left(s, M_{Q}^{2}, M_{q}^{2}\right)}{M_{Q}}}\right] .
\end{aligned}
$$

Some ordering of terms gives

$$
\begin{aligned}
\sigma & =-\frac{1}{3 \cdot 2^{10} \pi^{8} s}\left(\frac{\alpha}{\pi}\right)^{2} \int\left[\left|M_{B}\right|^{2} d \cos \theta_{q} d \phi_{q}\right] d M_{Q}^{2} \frac{d M_{q}^{2}}{M_{q}^{2}} \sqrt{1-\frac{4 \mu^{2}}{M_{q}^{2}}}\left(1+\frac{2 \mu^{2}}{M_{q}^{2}}\right) \sqrt{1-\frac{4 m^{2}}{M_{Q}^{2}}} \times \\
& \times \lambda^{\frac{1}{2}}\left(s, M_{Q}^{2}, M_{q}^{2}\right)\left[\frac{m^{2}}{M_{q}^{2} M_{Q}^{2}+\frac{m^{2}}{M_{Q}^{2}} \lambda\left(s, M_{Q}^{2}, M_{q}^{2}\right)}+\right. \\
& \left.+\frac{M_{Q}^{2}-2 m^{2}}{\left(s-M_{Q}^{2}-M_{q}^{2}\right) \sqrt{1-\frac{4 m^{2}}{M_{Q}^{2}}} \lambda^{\frac{1}{2}}\left(s, M_{Q}^{2}, M_{q}^{2}\right)} \ln \frac{s-M_{Q}^{2}-M_{q}^{2}-\sqrt{1-\frac{4 m^{2}}{M_{Q}^{2}}} \lambda^{\frac{1}{2}}\left(s, M_{Q}^{2}, M_{q}^{2}\right)}{s-M_{Q}^{2}-M_{q}^{2}+\sqrt{1-\frac{4 m^{2}}{M_{Q}^{2}}} \lambda^{\frac{1}{2}}\left(s, M_{Q}^{2}, M_{q}^{2}\right)}\right], \quad(23)
\end{aligned}
$$

or with explicit expression of Born separated (two body phase space is taken from formula (36) of ref. [29]):

$$
\begin{aligned}
\sigma & =\frac{1}{(2 \pi)^{6}} \int\left[\frac{1}{(2 \pi)^{2}} \cdot \frac{\lambda^{\frac{1}{2}}\left(1, \frac{m^{2}}{s}, \frac{m^{2}}{s}\right)}{8}\left|M_{B}\right|^{2} d \cos \theta_{q} d \phi_{q}\right] \times \lambda^{-\frac{1}{2}}\left(1, \frac{m^{2}}{s}, \frac{m^{2}}{s}\right) \\
& \times \frac{(-2)}{3 s}\left(\frac{\alpha}{\pi}\right)^{2} \int d M_{Q}^{2} \frac{d M_{q}^{2}}{M_{q}^{2}} \sqrt{1-\frac{4 \mu^{2}}{M_{q}^{2}}}\left(1+\frac{2 \mu^{2}}{M_{q}^{2}}\right) \times\left[\frac{m^{2} \sqrt{1-\frac{4 m^{2}}{M_{Q}^{2}}} \lambda^{\frac{1}{2}}\left(s, M_{Q}^{2}, M_{q}^{2}\right)}{M_{q}^{2} M_{Q}^{2}+\frac{m^{2}}{M_{Q}^{2}} \lambda\left(s, M_{Q}^{2}, M_{q}^{2}\right)}\right. \\
& \left.+\frac{M_{Q}^{2}-2 m^{2}}{s-M_{q}^{2}-M_{Q}^{2}} \ln \frac{s-M_{q}^{2}-M_{Q}^{2}-\sqrt{1-\frac{4 m^{2}}{M_{Q}^{2}}} \lambda^{\frac{1}{2}}\left(s, M_{Q}^{2}, M_{q}^{2}\right)}{s-M_{q}^{2}-M_{Q}^{2}+\sqrt{1-\frac{4 m^{2}}{M_{Q}^{2}}} \lambda^{\frac{1}{2}}\left(s, M_{Q}^{2}, M_{q}^{2}\right)}\right]
\end{aligned}
$$




\section{A.4 Result.}

From (24) we obtain analog of formula (5) of ref. [20]:

$$
\begin{aligned}
\widetilde{B}_{f} & =-\frac{2}{3 s}\left(\frac{\alpha}{\pi}\right)^{2} \int d M_{Q}^{2} \frac{d M_{q}^{2}}{M_{q}^{2}} \sqrt{1-\frac{4 \mu^{2}}{M_{q}^{2}}}\left(1+\frac{2 \mu^{2}}{M_{q}^{2}}\right)\left(\frac{m^{2} \sqrt{1-\frac{4 m^{2}}{M_{Q}^{2}}} \lambda^{\frac{1}{2}}\left(s, M_{Q}^{2}, M_{q}^{2}\right)}{M_{q}^{2} M_{Q}^{2}+\frac{m^{2}}{M_{Q}^{2}} \lambda\left(s, M_{Q}^{2}, M_{q}^{2}\right)}+\right. \\
& \left.+\frac{M_{Q}^{2}-2 m^{2}}{s-M_{q}^{2}-M_{Q}^{2}} \ln \frac{s-M_{q}^{2}-M_{Q}^{2}-\sqrt{1-\frac{4 m^{2}}{M_{Q}^{2}}} \lambda^{\frac{1}{2}}\left(s, M_{Q}^{2}, M_{q}^{2}\right)}{s-M_{q}^{2}-M_{Q}^{2}+\sqrt{1-\frac{4 m^{2}}{M_{Q}^{2}}} \lambda^{\frac{1}{2}}\left(s, M_{Q}^{2}, M_{q}^{2}\right)}\right)
\end{aligned}
$$

Note that the factor $\frac{1}{(2 \pi)^{6}}$ had to be dropped out to avoid double counting. This factor of phase space parametrization was already incorporated into the formula (17).

In order to make comparison with older calculations, we recall formula (5) of ref. [20]; case of $a=0$, which is exact for the emission of extra lepton pair from initial state.

$$
\begin{aligned}
\widetilde{B_{f}} & =-\frac{2}{3 s}\left(\frac{\alpha}{\pi}\right)^{2} \int d M_{Q}^{2} \frac{d M_{q}^{2}}{M_{q}^{2}} \sqrt{1-\frac{4 \mu^{2}}{M_{q}^{2}}}\left(1+\frac{2 \mu^{2}}{M_{q}^{2}}\right)\left(\frac{m^{2} \lambda^{\frac{1}{2}}\left(s, M_{Q}^{2}, M_{q}^{2}\right)}{M_{q}^{2} s+\frac{m^{2}}{s} \lambda\left(s, M_{Q}^{2}, M_{q}^{2}\right)}+\right. \\
& \left.+\frac{s-2 m^{2}}{\sqrt{1-\frac{4 m^{2}}{s}}\left(s+M_{q}^{2}-M_{Q}^{2}\right)} \ln \frac{s+M_{q}^{2}-M_{Q}^{2}-\sqrt{1-\frac{4 m^{2}}{s}} \lambda^{\frac{1}{2}}\left(s, M_{Q}^{2}, M_{q}^{2}\right)}{s+M_{q}^{2}-M_{Q}^{2}+\sqrt{1-\frac{4 m^{2}}{s}} \lambda^{\frac{1}{2}}\left(s, M_{Q}^{2}, M_{q}^{2}\right)}\right) .
\end{aligned}
$$

We have now collected all formulae necessary for numerical results. 


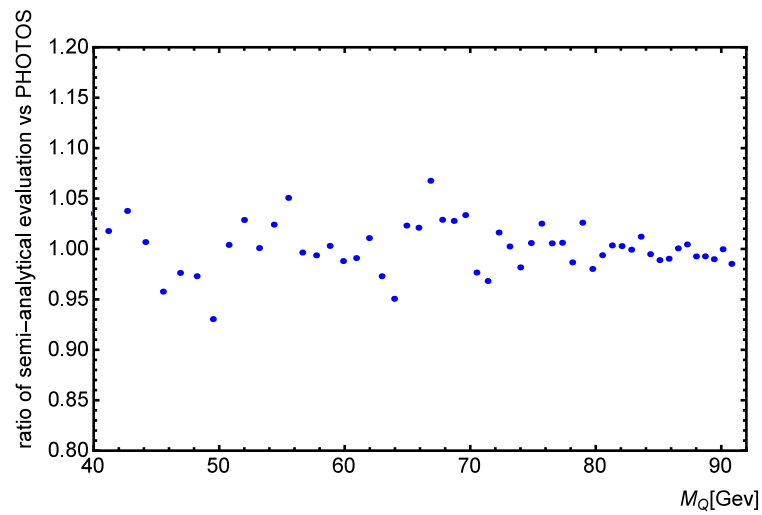

a) $p p \rightarrow Z \rightarrow e^{+} e^{-}\left(e^{+} e^{-}\right)$

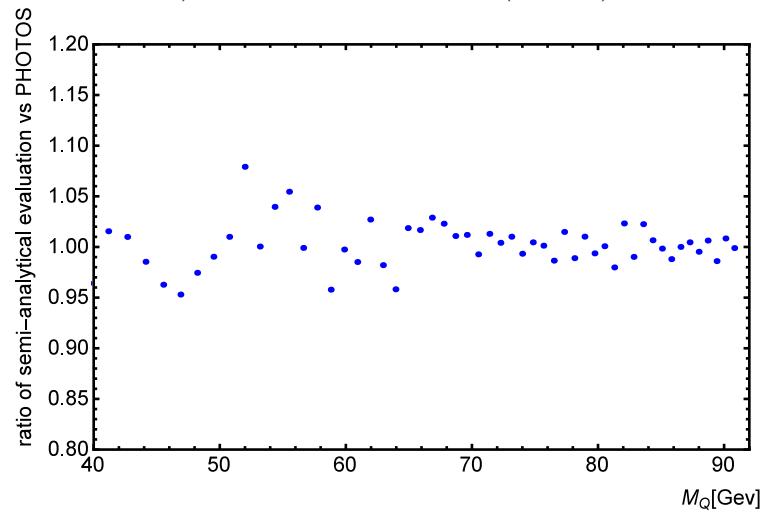

c) $p p \rightarrow Z \rightarrow \mu^{+} \mu^{-}\left(e^{+} e^{-}\right)$

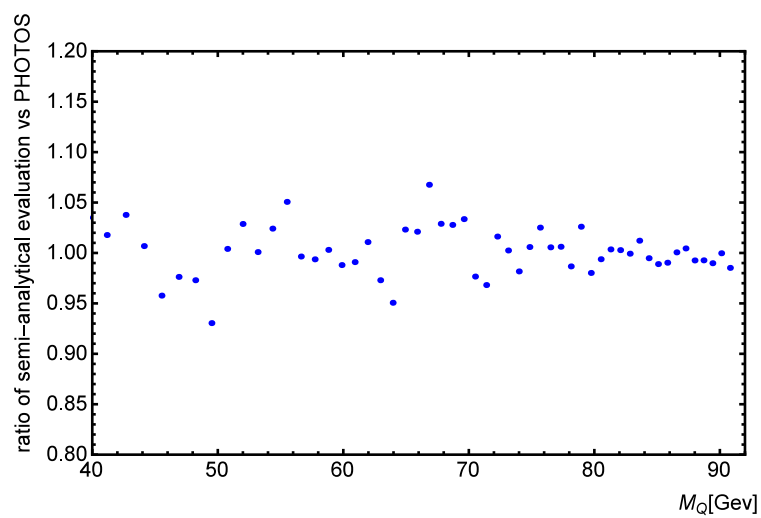

b) $p p \rightarrow Z \rightarrow e^{+} e^{-}\left(\mu^{+} \mu^{-}\right)$

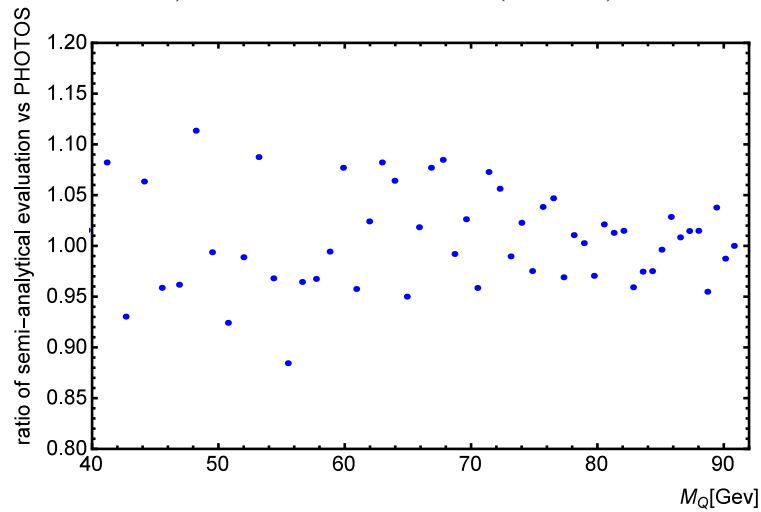

d) $p p \rightarrow Z \rightarrow \mu^{+} \mu^{-}\left(\mu^{+} \mu^{-}\right)$

Figure 11: Number of events from PYTHIA multiplied by a factor resulting from formula (25) divided by number of events from PYTHIA $\times$ PHOTOS. For these particular plots there is difference in PYTHIA initialization parameters; energy range of leptonic system is limited to $[91.183,91.252] \mathrm{GeV}$ window. 
WeakSingleBoson:ffbar2gmZ $=$ on 23: onMode $=$ off

$23:$ onIfAny $=11$

$23: \mathrm{mMin}=10.0$

$23: \operatorname{mMax}=200.0$

HadronLevel: Hadronize $=$ off

SpaceShower:QEDshowerByL = off

SpaceShower: QEDshowerByQ = off

PartonLevel:ISR $=$ off

PartonLevel:FSR $=$ off

Beams:idA $=2212$

Beams:idB $=2212$

Beams: $\mathrm{eCM}=14000.0$

a) $p p \rightarrow Z \rightarrow e^{+} e^{-}\left(e^{+} e^{-}, \mu^{+} \mu^{-}\right)$

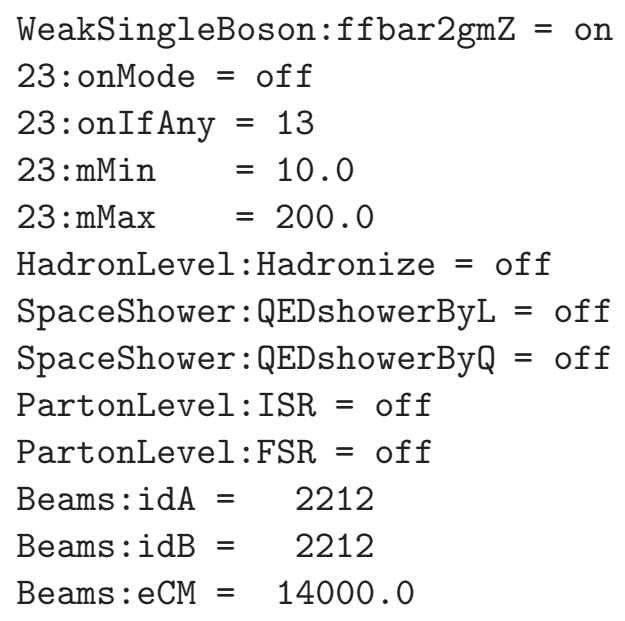

b) $p p \rightarrow Z \rightarrow \mu^{+} \mu^{-}\left(e^{+} e^{-}, \mu^{+} \mu^{-}\right)$

Figure 12: Initialization parameters for PYTHIA. 